\title{
QUANTUM TRAJECTORIES, FEEDBACK AND SQUEEZING
}

\author{
A. Barchielli, M. Gregoratti, M. Licciardo \\ Dipartimento di Matematica, Politecnico di Milano, \\ Piazza Leonardo da Vinci 32, I-20133, Milano, Italy
}

October 28, 2018

\begin{abstract}
Quantum trajectory theory is the best mathematical set up to model continual observations of a quantum system and feedback based on the observed output. Inside this framework, we study how to enhance the squeezing of the fluorescence light emitted by a two-level atom, stimulated by a coherent monochromatic laser. In the presence of a Wiseman-Milburn feedback scheme, based on the homodyne detection of a fraction of the emitted light, we analyze the squeezing dependence on the various control parameters.
\end{abstract}

\section{Introduction}

Photo-detection theory in continuous time has been widely developed [1, 2, 3, [4, 5] and applied, in particular, to the fluorescence light emitted by a two-level atom stimulated by a coherent monochromatic laser [6, 5. As well as various feedback schemes on the atom evolution, based on the outcoming photocurrent, have been proposed [7, 8, 13. However the introduction and the analysis of feedback have always been focused on the control of the atom dynamics. The typical aim was to drive the atom to a preassigned asymptotic state or to a preassigned asymptotic unitary dynamics $9,10,11,12,13$.

Here, on the contrary, we are interested in the emitted light and in employing control and feedback processes to enhance its squeezing properties. These can be checked by homodyne detection and spectral analysis of the output current. For these reasons we consider the mathematical description of photo-detection based on classical stochastic differential equations (quantum trajectories), as it is suitable both to consistently compute the homodyne spectrum of fluorescence light, and to introduce feedback and control in the mathematical formulation. We study how the squeezing depends on the various control parameters and how feedback mechanisms can be successfully introduced. We consider only Markovian feedback schemes à la Wiseman-Milburn [7, 8, as they leave the homodyne spectrum explicitly computable. 


\section{Detection and feedback scheme}

Consider a two-level atom with Hilbert space $\mathcal{H}=\mathbb{C}^{2}$ and lowering and rising operators $\sigma_{-}$and $\sigma_{+}$. Let the Pauli matrices be $\sigma_{x}=\sigma_{-}+\sigma_{+}, \sigma_{y}=\mathrm{i}\left(\sigma_{-}-\sigma_{+}\right)$, $\sigma_{z}=\sigma_{+} \sigma_{-}-\sigma_{-} \sigma_{+}$and let the vector of operators $\left(\sigma_{x}, \sigma_{y}, \sigma_{z}\right)$ be denoted by $\vec{\sigma}$. Let also the eigenprojectors of $\sigma_{z}$ be denoted by $P_{+}=\sigma_{+} \sigma_{-}$and $P_{-}=\sigma_{-} \sigma_{+}$ and, for every angle $\phi$, let us introduce the unitary selfadjoint operator

$$
\sigma_{\phi}=\mathrm{e}^{\mathrm{i} \phi} \sigma_{-}+\mathrm{e}^{-\mathrm{i} \phi} \sigma_{+}=\cos \phi \sigma_{x}+\sin \phi \sigma_{y} .
$$

A state $\rho$ of the atom is represented by a point $\vec{x}$ in the Bloch sphere,

$$
\rho=\frac{1}{2}(1+\vec{x} \cdot \vec{\sigma}), \quad \vec{x} \in \mathbb{R}^{3}, \quad|\vec{x}| \leq 1 .
$$

We admit an open Markovian evolution for the atom, subjected to "dephasing" effects and to interactions both with a thermal bath and, via absorption and emission of photons, with the electromagnetic field. In particular we suppose that the atom is stimulated by a coherent monochromatic laser and that the emitted light is partially lost in the so called forward channel and partially gathered in two so called side channels for homodyne detection.

Let the free Hamiltonian of the atom be $\omega_{0} \sigma_{z} / 2, \omega_{0}>0$. Let the natural linewidth of the atom be $\gamma$, let the intensities of the dephasing and thermal effects be given by the adimensional parameters $k_{\mathrm{d}} \geq 0$ and $\bar{n} \geq 0$, let the stimulating laser have frequency $\omega>0$ and Rabi frequency $\Omega \geq 0$. Let $\Delta \omega=\omega_{0}-\omega$ denote the detuning.

Let the fractions of light emitted in the forward and in the two side channels be $\left|\alpha_{0}\right|^{2},\left|\alpha_{1}\right|^{2},\left|\alpha_{2}\right|^{2}$ respectively $\left(\left|\alpha_{0}\right|^{2}+\left|\alpha_{1}\right|^{2}+\left|\alpha_{2}\right|^{2}=1,\left|\alpha_{0}\right|^{2}>0,\left|\alpha_{1}\right|^{2}>0\right.$, $\left|\alpha_{2}\right|^{2} \geq 0$ ), and, for the side channels, let the initial phase of the local oscillator in the corresponding detector be $\vartheta_{k}=\arg \alpha_{k}, k=1,2$. Changing $\vartheta_{k}$ means to change the measuring apparatus. Let the two homodyne photocurrents be $I_{1}$ and $I_{2}$.

We introduce a feedback control scheme à la Wiseman-Milburn based on the photocurrent $I_{1}$ revealed in the side channel 1. Assuming instantaneous feedback, we modify the amplitude of the laser driving the atom by adding a term $g \mathrm{e}^{-\mathrm{i} \omega t} I_{1}(t) / \sqrt{\gamma}$ proportional to $I_{1}$, with the same frequency $\omega$ and with initial phase possibly different from that of the original laser. Let this difference be $\varphi$.

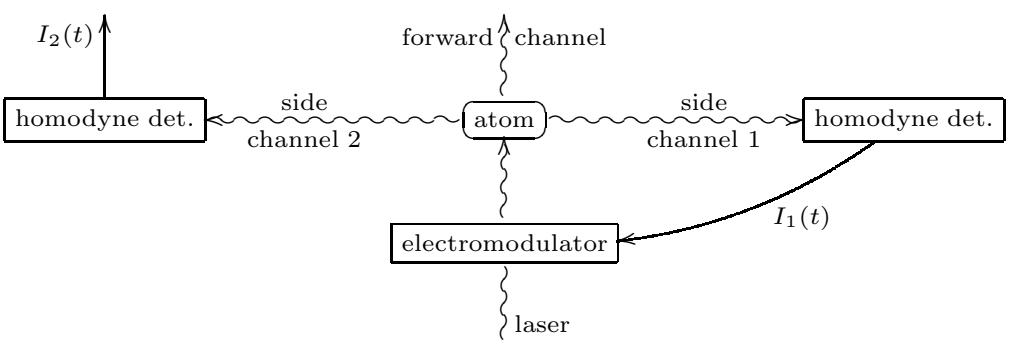


Then the atom has a Markovian evolution, whether we condition its state on continuous monitoring of the photocurrents, or we do not. Let us call $a$ priori state $\eta_{t}$ the unconditioned one and let us call a posteriori state $\rho_{t}$ the conditioned one. Of course $\eta_{t}$ is the mean of $\rho_{t}$. Let us write the evolution equations in the rotating frame, where they result to be time-homogeneous. Let us introduce first the parameters

$$
c=|g|\left|\alpha_{0}\right| / \sqrt{\gamma} \geq 0, \quad \Delta \omega_{c}=\Delta \omega+c \gamma\left|\alpha_{1}\right| \cos \left(\vartheta_{1}-\varphi\right) \in \mathbb{R} .
$$

The a priori state $\eta_{t}$ is governed by the Master equation

$$
\begin{gathered}
\mathrm{d} \eta_{t}=\mathcal{L} \eta_{t} \mathrm{~d} t \\
\mathcal{L} \rho=-\mathrm{i}\left[\frac{\Delta \omega_{c}}{2} \sigma_{z}+\frac{\Omega}{2} \sigma_{x}, \rho\right]+\gamma k_{\mathrm{d}}\left(\sigma_{z} \rho \sigma_{z}-\rho\right)+\gamma \bar{n}\left(\sigma_{+} \rho \sigma_{-}-\frac{1}{2}\left\{P_{-}, \rho\right\}\right) \\
+\gamma\left(\bar{n}+1-\left|\alpha_{1}\right|^{2}\right)\left(\sigma_{-} \rho \sigma_{+}-\frac{1}{2}\left\{P_{+}, \rho\right\}\right) \\
+\gamma\left(\alpha_{1} \sigma_{-}-\mathrm{i} c \sigma_{\varphi}\right) \rho\left(\bar{\alpha}_{1} \sigma_{+}+\mathrm{i} c \sigma_{\varphi}\right)-\frac{\gamma}{2}\left\{\left(\left|\alpha_{1}\right|^{2}-2 c\left|\alpha_{1}\right| \sin \left(\vartheta_{1}-\varphi\right)\right) P_{+}+c^{2}, \rho\right\} .
\end{gathered}
$$

The a posteriori state $\rho_{t}$ is governed by the non-linear stochastic Master equation

$$
\mathrm{d} \rho_{t}=\mathcal{L} \rho_{t} \mathrm{~d} t+\sqrt{\gamma} \mathcal{D}\left[\alpha_{1} \sigma_{-}-\mathrm{i} c \sigma_{\varphi}\right] \rho_{t} \mathrm{~d} W_{1}(t)+\sqrt{\gamma} \mathcal{D}\left[\alpha_{2} \sigma_{-}\right] \rho_{t} \mathrm{~d} W_{2}(t),
$$

where, for every matrix $a$, the superoperator $\mathcal{D}[a]$ is

$$
\mathcal{D}[a] \rho=a \rho+\rho a^{*}-\rho \operatorname{Tr}\left[\left(a+a^{*}\right) \rho\right],
$$

and where $W_{1}$ and $W_{2}$ are two independent Wiener processes. The two homodyne photocurrents are given by the generalized stochastic processes

$$
I_{k}(t)=\sqrt{\gamma}\left|\alpha_{k}\right| \operatorname{Tr}\left[\sigma_{\vartheta_{k}} \rho_{t}\right]+\dot{W}_{k}(t) .
$$

Note that each signal term $\sqrt{\gamma}\left|\alpha_{k}\right| \operatorname{Tr}\left[\sigma_{\vartheta_{k}} \rho_{t}\right]$ depends on the dynamics of the a posteriori state $\rho_{t}$ and that, typically, it is correlated to both the noise terms $\dot{W}_{1}(t)$ and $\dot{W}_{2}(t)$.

Let us remark that, even if the feedback is based on the singular stochastic process $I_{1}$, the mathematical formulation of the model is not affected by this singularity, as we do not observe directly the light in the forward channel.

We suppose that $\left|\alpha_{0}\right|$ is assigned by experimental constraints and that the control parameters are $\Omega, \Delta \omega,\left|\alpha_{1}\right|,\left|\alpha_{2}\right|, \vartheta_{1}, \vartheta_{2}, c$ and $\varphi$. Of course, if $c=0$, then there is no feedback action on the atom, so that its a priori dynamics is independent of the measurement process, that is of the fractions $\left|\alpha_{1}\right|^{2},\left|\alpha_{2}\right|^{2}$ and of the phases $\vartheta_{1}, \vartheta_{2}, \varphi$. On the contrary, if $c>0$, then the a priori dynamics is modified by the feedback loop and it depends also on $\left|\alpha_{1}\right|, \vartheta_{1}, c$ and $\varphi$. 


\section{Homodyne incoherent spectrum and squeez- ing}

We are interested in the light emitted by the atom and in particular in the squeezing properties of the light in the side channels 1 and 2. With the help of the incoherent spectrum of the homodyne photocurrents we can analyze the squeezing properties of the light detected in the two side channels, and thus we can investigate the effect of the control parameters.

When $\left|\alpha_{2}\right|^{2}=0$, the fluorescence light which is not lost in the forward channel is gathered in a unique side channel, so that the squeezing is analyzed just for that light which is also detected for the feedback loop. This means that the eventually squeezed light would not be available for other purposes. Thus in this case a unique homodyne detector is employed and $\left|\alpha_{1}\right|^{2}$ is its efficiency. The meaning and the possible usefulness of the squeezing of the light involved in the feedback loop is discussed by Wiseman [10. When $\left|\alpha_{2}\right|^{2}>0$, the fluorescence light which is not lost in the forward channel is split in the two side channels. The homodyne detection of the light in channel 1 allows both the analysis of its squeezing and the feedback control. The light in channel 2 is detected for squeezing analysis, as well as it could be employed for different uses. Let us remark that, even if we were interested in squeezing only for channel 2 , the choice of a feedback scheme based on homodyne detection in channel 1 would be still essential in order to get a time-homogeneous atomic evolution in the rotating frame.

The structure of $\mathcal{L}$ guarantees that, for every initial preparation of the atom, the a priori state $\eta_{t}$ asymptotically reaches the stationary state

$$
\rho_{\mathrm{eq}}=\frac{1}{2}\left(1+\vec{x}_{\mathrm{eq}} \cdot \vec{\sigma}\right), \quad \vec{x}_{\mathrm{eq}}=-\gamma\left(1-2 c\left|\alpha_{1}\right| \sin \left(\vartheta_{1}-\varphi\right)\right) A^{-1}\left(\begin{array}{l}
0 \\
0 \\
1
\end{array}\right),
$$

where $A$ is the $3 \times 3$ matrix giving $\mathcal{L}$ in the Bloch sphere language,

$$
\begin{gathered}
A=\left(\begin{array}{ccc}
a_{11} & a_{12} & 0 \\
a_{21} & a_{22} & \Omega \\
0 & -\Omega & a_{33}
\end{array}\right), \\
a_{11}=\gamma\left(\frac{1}{2}+\bar{n}+2 k_{\mathrm{d}}+2 c\left|\alpha_{1}\right| \cos \vartheta_{1} \sin \varphi+2 c^{2} \sin ^{2} \varphi\right), \\
a_{12}=\Delta \omega_{c}-\gamma\left(c\left|\alpha_{1}\right| \cos \left(\vartheta_{1}+\varphi\right)-2 c^{2} \sin 2 \varphi\right), \\
a_{21}=-\Delta \omega_{c}-\gamma\left(c\left|\alpha_{1}\right| \cos \left(\vartheta_{1}+\varphi\right)-2 c^{2} \sin 2 \varphi\right), \\
a_{22}=\gamma\left(\frac{1}{2}+\bar{n}+2 k_{\mathrm{d}}-2 c\left|\alpha_{1}\right| \sin \vartheta_{1} \cos \varphi+2 c^{2} \cos ^{2} \varphi\right), \\
a_{33}=\gamma\left(1+2 \bar{n}-2 c\left|\alpha_{1}\right| \sin \left(\vartheta_{1}-\varphi\right)+2 c^{2}\right) .
\end{gathered}
$$

Thus we can introduce the homodyne incoherent spectrum of the light revealed in each side channel $k$ as the limit of the normalized variance of the Fourier 
transform of the photocurrent $I_{k}$

$$
S_{k}(\mu)=\lim _{T \rightarrow+\infty} \frac{1}{T}\left\{\mathbb{E}\left[\left|\int_{0}^{T} \mathrm{e}^{\mathrm{i} \mu s} I_{k}(s) \mathrm{d} s\right|^{2}\right]-\left|\mathbb{E}\left[\int_{0}^{T} \mathrm{e}^{\mathrm{i} \mu s} I_{k}(s) \mathrm{d} s\right]\right|^{2}\right\} .
$$

It is a positive even function of its real argument $\mu$ which can be computed from equations (11) and (2) by Ito calculus and by the full theory of Quantum Continual Measurement, which can provide the first and second moments of $I_{1}$ [2, 5]. Thus, for every initial state of the atom, we can obtain

$$
S_{k}(\mu)=1+2 \gamma\left|\alpha_{k}\right|^{2}\left(\frac{A}{A^{2}+\mu^{2}} \vec{t}_{k}\right) \cdot \vec{s},
$$

where $\vec{t}_{k}$ and $\vec{s}$ are the vectors in $\mathbb{R}^{3}$ defined as

$$
\begin{gathered}
\vec{t}_{1}=\operatorname{Tr}\left[\left(\mathrm{e}^{\mathrm{i} \vartheta_{1}} \sigma_{-} \rho_{\mathrm{eq}}+\mathrm{e}^{-\mathrm{i} \vartheta_{1}} \rho_{\mathrm{eq}} \sigma_{+}-\operatorname{Tr}\left[\sigma_{\vartheta_{1}} \rho_{\mathrm{eq}}\right] \rho_{\mathrm{eq}}+\mathrm{i} \frac{c}{\left|\alpha_{1}\right|}\left[\rho_{\mathrm{eq}}, \sigma_{\varphi}\right]\right) \vec{\sigma}\right], \\
\vec{t}_{2}=\operatorname{Tr}\left[\left(\mathrm{e}^{\mathrm{i} \vartheta_{2}} \sigma_{-} \rho_{\mathrm{eq}}+\rho_{\mathrm{eq}} \mathrm{e}^{-\mathrm{i} \vartheta_{2}} \sigma_{+}-\operatorname{Tr}\left[\sigma_{\vartheta_{2}} \rho_{\mathrm{eq}}\right] \rho_{\mathrm{eq}}\right) \vec{\sigma}\right], \\
\vec{s}=\left(\begin{array}{c}
\cos \vartheta_{k} \\
\sin \vartheta_{k} \\
0
\end{array}\right) .
\end{gathered}
$$

More explicitly, by using the Bloch components of the equilibrium state (3), we get

$$
\begin{gathered}
\vec{t}_{1}=\left(\begin{array}{c}
\left(1+z_{\mathrm{eq}}-x_{\mathrm{eq}}^{2}\right) \cos \vartheta_{1}-x_{\mathrm{eq}} y_{\mathrm{eq}} \sin \vartheta_{1} \\
\left(1+z_{\mathrm{eq}}-y_{\mathrm{eq}}^{2}\right) \sin \vartheta_{1}-x_{\mathrm{eq}} y_{\mathrm{eq}} \cos \vartheta_{1} \\
-\left(1+z_{\mathrm{eq}}\right)\left(x_{\mathrm{eq}} \cos \vartheta_{1}+y_{\mathrm{eq}} \sin \vartheta_{1}\right)
\end{array}\right)+\frac{2 c}{\left|\alpha_{1}\right|}\left(\begin{array}{c}
z_{\mathrm{eq}} \sin \varphi \\
-z_{\mathrm{eq}} \cos \varphi \\
-x_{\mathrm{eq}} \sin \varphi+y_{\mathrm{eq}} \cos \varphi
\end{array}\right) \\
\vec{t}_{2}=\left(\begin{array}{c}
\left(1+z_{\mathrm{eq}}-x_{\mathrm{eq}}^{2}\right) \cos \vartheta_{2}-x_{\mathrm{eq}} y_{\mathrm{eq}} \sin \vartheta_{2} \\
\left(1+z_{\mathrm{eq}}-y_{\mathrm{eq}}^{2}\right) \sin \vartheta_{2}-x_{\mathrm{eq}} y_{\mathrm{eq}} \cos \vartheta_{2} \\
-\left(1+z_{\mathrm{eq}}\right)\left(x_{\mathrm{eq}} \cos \vartheta_{2}+y_{\mathrm{eq}} \sin \vartheta_{2}\right)
\end{array}\right)
\end{gathered}
$$

Each spectrum $S_{k}$ depends on $k_{\mathrm{d}}, \bar{n}, \Omega, \Delta \omega,\left|\alpha_{k}\right|, \vartheta_{k}, c$ and $\varphi$. Moreover, $S_{2}$ depends also on $\left|\alpha_{1}\right|$ and $\vartheta_{1}$.

If $S_{k}(\mu)<1$ for some $\mu$ and $\vartheta_{k}$, then the homodyne detection identified by $\vartheta_{k}$ reveals a squeezed mode around $\mu$ of the light in channel $k$.

Independently of the presence of the feedback loop, every time a parameter $\left|\alpha_{k}\right|$ vanishes, the corresponding photocurrent $I_{k}$ reduces to a pure white noise (shot noise due to the local oscillator) with spectrum $S_{k}=1$ for every choice of the other parameters.

Analyze first $c=0$, the situation without feedback. In this case each dependence on $\varphi$ disappears and $S_{2}$ becomes independent of $\left|\alpha_{1}\right|$ and $\vartheta_{1}$, so that there is no difference between $S_{1}$ and $S_{2}$. Moreover, the dependence of each spectrum $S_{k}$ on the corresponding $\left|\alpha_{k}\right|$ reduces to the explicit multiplication coefficient in (4). Therefore, when the control parameters $\Omega$ and $\Delta \omega$ give squeezed light 
in channel $k$, the lowering of $S_{k}$ under the shot noise level is anyhow directly proportional to the fraction of emitted light gathered in that channel.

For $\Omega=0$ and $\bar{n}=0$ there is no fluorescence light in the long run, so that each photocurrent $I_{k}$ again reduces to a pure white noise with spectrum $S_{k}=1$.

For $\Omega=0$ and $\bar{n}>0$ there is no dependence on $\vartheta_{k}$ and $S_{k}>1$. In this case there is only thermal light with carrier frequency $\omega_{0}$, while the local oscillator is at frequency $\omega$. The result are two temperature dependent Lorentzian peaks at $\mu= \pm \Delta \omega$. The white noise contribution is always present.

When $\Omega>0, S_{k}$ becomes $\vartheta_{k}$-dependent and it can go below the shot noise level. This fact means that some negative correlation between signal and noise has been developed. Some examples are plotted for both channels, always for $\gamma=1, k_{\mathrm{d}}=0, \bar{n}=0$ and $\left|\alpha_{1}\right|^{2}=\left|\alpha_{2}\right|^{2}=0.45$. Figures 1 and 2 show $S_{1}$ and $S_{2}$ respectively for $\Delta \omega=0$ (line 1) and for $\Delta \omega=-2$ (line 3), every time for values of $\Omega$ and $\vartheta_{k}$ chosen in order to have a region with a pronounced squeezing $\left(\Omega=0.2976, \vartheta_{k}=-\pi / 2\right.$ for line $1 ; \Omega=2.0526, \vartheta_{k}=0.1449$ for line 3$)$.

One could also compare the homodyne spectrum with and without $\bar{n}$ and $k_{\mathrm{d}}$, thus verifying that the squeezing is very sensitive to any small perturbation.

Allow now $c \geq 0$. The optimal squeezing in channel 1 is always found for $\Omega^{2}=0$ and the feedback loop is very helpful, giving good visible minima of $S_{1}$ also when $\left|\alpha_{1}\right|^{2}$ is not close to 1 . For example, in the case $\left|\alpha_{1}\right|^{2}=0.45$, Fig. 1 shows $S_{1}$ for $\Delta \omega=0$ (line 2) and for $\Delta \omega=-2$ (line 4), every time for values of $\Omega, \vartheta_{1}, c$ and $\varphi$ chosen in order to enhance the squeezing $(\Omega=0, c=0.2936$, $\varphi-\vartheta_{1}=\pi / 2$ for line $2 ; \Omega=0, c=0.3762, \vartheta_{1}=0.0482, \varphi=1.9941$ for line 4). Again $\gamma=1, k_{\mathrm{d}}=0, \bar{n}=0$. The utility of the feedback scheme can be appreciated by comparing lines 1 and 3 with lines 2 and 4 respectively.

If we are interested in the light emitted in channel 2 and if $\left|\alpha_{1}\right|^{2}$ and $\left|\alpha_{2}\right|^{2}$ are assigned by some constraints, then the squeezing in channel 2 can be enhanced by a feedback scheme based on the photocurrent coming from channel 1, but the feedback performance is not as good as it can be for the squeezing in channel 1 itself. Fig. 2 shows $S_{2}$ for $\Delta \omega=0$ (line 2) and for $\Delta \omega=-2$ (line 4 ), every time for values of $\Omega, \vartheta_{1}, c, \varphi$ and $\vartheta_{2}$, chosen in order to enhance the squeezing

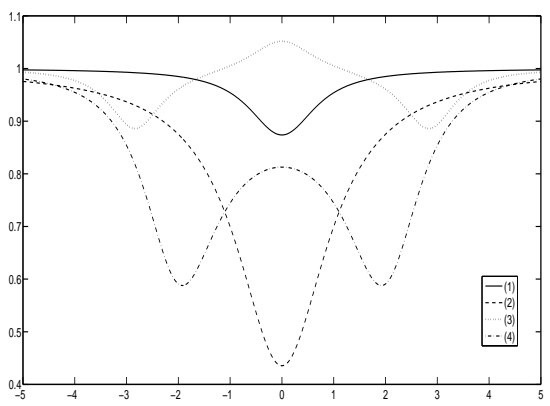

Figure 1: Channel 1

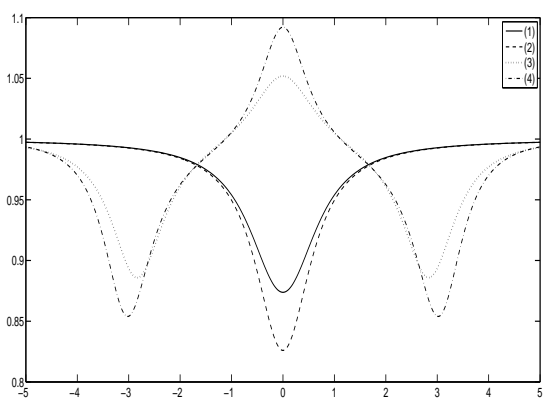

Figure 2: Channel 2 
$\left(\Omega=0.2698, \vartheta_{1}=\pi / 2, c=0.0896, \varphi=0, \vartheta_{2}=\pi / 2\right.$ for line $2 ; \Omega=2.329$, $\vartheta_{1}=0.2896, c=0.1346, \varphi=-1.2902, \vartheta_{2}=0.0728$ for line 4$)$.

Anyway, if the only constraint is $\left|\alpha_{1}\right|^{2}+\left|\alpha_{2}\right|^{2}=1-\left|\alpha_{0}\right|^{2}$ and we are free in the choice of $\left|\alpha_{1}\right|^{2}$ and $\left|\alpha_{2}\right|^{2}$, then the best observable squeezing in channel 2 is obtained in the limit case $\left|\alpha_{1}\right|^{2}=0, c=0$. That is, when the whole non-lost light is gathered just in channel 2 and the white noise $I_{1}$ revealed in channel 1 is ignored.

Let us remark that, when we use control parameters enhancing the squeezing for channel $k$, every time $\Delta \omega=0$ we find the spectrum $S_{k}$ with an absolute minimum in $\mu=0$, while whenever $\Delta \omega \neq 0$ we find the spectrum $S_{k}$ with two absolute minima, symmetric with respect to $\mu=0$, which turns out to be a local maximum.

Finally let us remark that the idea of the papers [11, 12, 13, was to choose the control parameters in such a way that, in the rotating frame, the atom is frozen in a preassigned pure state $h_{0} \in \mathcal{H}$, i.e. in such a way that, in the rotating frame, both the a priori state $\eta_{t}$ and the a posteriori state $\rho_{t}$ asymptotically reach $\rho_{\text {eq }}=\left|h_{0}\right\rangle\left\langle h_{0}\right|$. This is possible in an exact way only in a very ideal case, which in our notations corresponds to $\Delta \omega=0, \varphi=0, \vartheta_{1}= \pm \pi / 2,\left|\alpha_{1}\right|=1$, $\alpha_{0}=\alpha_{2}=0, k_{\mathrm{d}}=0, \bar{n}=0$, which implies in particular $a_{12}=a_{21}=0$ and $x_{\mathrm{eq}}=0$. Then, the a posteriori state $\rho_{t}$ is driven to a pure given state if $\Omega$ and $c$ are such that $y_{\text {eq }}^{2}+z_{\text {eq }}^{2}=1$ and $2 c \sin \vartheta_{1}=1+z_{\text {eq. }}$. But this implies $\vec{t}_{1}=0$ and the two incoherent spectra reduce to pure shot noise. This is reasonable: if the atom if frozen there is not incoherent scattering of light. One can check that actually only the coherent scattering survives, giving a $\delta$-contribution in $\mu=0$

to the complete spectrum. If the freezing of the atom is only approximate, or if one tries to maximize the atomic squeezing (which is another way of stopping the atomic motion), one can check that all the spectra tend to become flatter and the squeezing tends to disappear.

\section{References}

[1] E. B. Davies, Quantum Theory of Open Systems (Academic Press, London, 1976).

[2] A. Barchielli, Quantum Opt. 2 (1990) 423-441.

[3] A. Barchielli, V. P. Belavkin, J. Phys. A: Math. Gen. 24 (1991) 1495-1514.

[4] A. Barchielli, A. M. Paganoni, Quantum Semiclass. Opt. 8 (1996) 133-156.

[5] A. Barchielli, Continual Measurements in Quantum Mechanics and Quantum Stochastic Calculus, in Open Quantum Systems III, eds. S. Attal, A. Joye, C.-A. Pillet, Lecture Notes in Mathematics 1882 (Springer, Berlin, 2006), pp. 207-291.

[6] H. M. Wiseman, G. J. Milburn, Phys. Rev. A 47 (1993) 1652-1666.

[7] H. M. Wiseman, G. J. Milburn, Phys. Rev. Lett. 70 (1993) 548-551. 
[8] H. M. Wiseman, Phys. Rev. A 49 (1994) 2133-2150.

[9] D. F. Walls, G. J. Milburn, Quantum Optics (Springer, Berlin 1994).

[10] H. M. Wiseman, Phys. Rev. Lett. 81 (1998) 3840-3843.

[11] J. Wang, H. M. Wiseman, Phys. Rev. A 64 (2001) 063810.

[12] J. Wang, H. M. Wiseman, G. J. Milburn, Chemical Physics 268 (2001) 221-235.

[13] H. M. Wiseman, S. Mancini, J. Wang, Phys. Rev. A 66 (2002) 013807. 